\title{
ISO 15189 accreditation experience at the Microbiology Laboratory of a Turkish University Hospital
}

\author{
Yesim Gurol*, Emine Kurt, Iskender Karalti, Yasemin Ozturk, Zuhal Tekkanat Tazegun, \\ Selami Sozubir, Gulden Yilmaz and Ayca Vitrinel \\ Yeditepe University Hospital, Devlet Yolu Ankara cad. No: 102-104 34752, Kozyatagi-Istanbul,Turkey.
}

Accepted 22 August, 2011

\begin{abstract}
We aimed to share the experience of the ISO/IEC 15189:2007 accreditation process in a University Hospital's Medical Microbiology Laboratory before and after the assessment, and tried to seek answers to some questions in applying the standard. Our ISO 15189 accreditation process began in 2008 after having acquired $\mathrm{JCl}$ accreditation in 2007. The aim was to be the first accreditated university hospital laboratory in our country. The applied tests in bacteriology, mycology, parasitology, virology, immunology and molecular serology were accreditated. We completed the validation and verification procedures mostly applied to quantitative analyses. Regarding the external quality controls and the internal controls, the corrective actions provided us great control and self correction. We increased the rate of success in the quality controls. We encouraged our laboratory personnel to fill out a laboratory discrepancy form to control our process and to arrange training. During the course of 2009, 60 of the 85 forms were completed in the microbiology laboratory among the central laboratories. Statistical analyses were performed throughout a year and this information helped us in making improvements. The number of training hours in one year was found as $78 \mathrm{~h}$ and $\mathbf{3 0}$ min during the routine laboratory process. We should provide support in bringing quality to laboratories in our country. As a matter of fact, having our own external quality controls is believed to bring cost effectiveness in acquiring an accreditation process.
\end{abstract}

Key words: Accreditation, microbiology, laboratories.

\section{INTRODUCTION}

Laboratories should go through some processes in order to yield reliable results. These processes are maintained under the term of quality standards and supported by many organizations and laboratories in accordance to guidelines of the ministry of health in the related country.

Clinical microbiology is mostly based on interpretation while giving the results. There may be laboratory errors, which can lead to problems in the process. Such errors may occur in the pre-analytical, analytical, and postanalytical steps and influence the therapy of the patient, in addition to misleading the clinician (Amsterdam et al., 2004).

Accreditation is a procedure by which an authoritative body gives formal recognition that a body or person is

${ }^{\star}$ Corresponding author. E-mail: yesimg@yeditepe.edu.tr. Tel: 902165780267. Fax :902165780575. competent to carry out specific tasks. Accreditation in clinical and medical laboratories is a process that is acquired by and supported by laboratories in several countries. The international standard for medical laboratories is a must for several purposes, such as interpretation of results, education, training, etc (Kenny, 2001).

Basically, the accreditation process is based on the ISO/IEC 15189:2007 standard (ISO 15189 Standards, 2007). This standard has chapters including quality policy, staff education and training, quality assurance, document control, records, maintanence, archiving, accommodation and environment, instruments, reagents and consumables management, safety, environmental aspects, research and development, list of examination procedures, request protocols, primary sample, collection and handling of laboratory samples, quality control, laboratory information system, reporting results, remedial actions and handling of complaints, internal audits, and 
interaction with patients and health professionals.

The Central Laboratories of Yeditepe University Hospitals and Affiliated Institutions began to provide services together with the opening of the Yeditepe University Hospital on 26 August 2005. It has an advanced infrastructure and is equipped with universally accepted laboratory technologies. The accepted main policy of the Central Laboratories of Yeditepe University Hospitals and Affiliated Institutions is supplying laboratory services in compliance with national and international standards and test methods at a possible minimum time and based on accuracy, subjectivity, independence, honesty, confidentiality and reliability principles, and to contribute to and continue improvement of the medical science with training and research studies.

\section{MATERIALS AND METHODS}

The Yeditepe University Hospital Microbiology Laboratory applies tests in bacteriology, mycology, parasitology, virology, immunology and molecular serology. Our ISO 15189 accreditation process began in 2008 after having obtained $\mathrm{JCl}$ accreditation in 2007 . The aim was to be the first accreditated university hospital laboratory in our country. Providing all the requirements in the standard, we tried to manage requirements such as verification and validation procedures.

The main principles of the established quality system and how the requirements of the standard were met were documented by a Quality Handbook and procedures that were referred in the Quality Handbook. The Quality handbook aims at making a general summary of the Quality Management System and to make it understood, applied and continued at each level.

While the Yeditepe University Hospitals and Affiliated Institutions are establishing the quality managements system, the following are undertaken;

1. The quality management defines the whole procedures that have an impact on operation of activities that are related to quality,

2. The relationship between defined processes and sub-processes are determined,

3. Necessary methods and evaluation techniques are determined in order to carry out processes under effective and controlled conditions,

4. Analysis, tracking and measurement of processes are made, necessary activities are determined, and put into operation in order to reach planned results and thrive for continuous improvement

\section{Before the assessment}

Taking into consideration that the regulations of every article in the standard was already accomplished, they should all have been checked. So we decided to make an internal assessment with a checklist. After observing some defects and failure to fulfilment, they were completed as soon as possible. Before the date of the assessment, the lab personnel was also educated according to the checklist.

\section{RESULTS}

\section{An overview of the assessment}

During the assessment, in bacteriology, the culture and staining methods were the the first articles to be checked. The internal daily quality controls of Gram and acid-fast staining and weekly control of the disk diffusion tests with ATCC references were the first internal quality controls to be checked. The agar and liquid medium in every different lot number were also evaluated. The liquid medium beside the agar medium in every type of culture was questioned. Beside some selective agar, the use of classical media was also recommended.

The anaerobic culture interpretation and period of incubation was also a point of question. The algorithms and the plans of staining and cultivation were carefully considered.

In the mycobacteriology laboratory, the location of the laboratory, the kind of laminair safety cabin, the waste boxes, the disinfectants, the centrifuge, and the incubator were markedly noted in the report. In the test document, we were recommended to make a record of the sample collection, especially for sputum in details, such as not washing the mouth with tap water in order to prevent nonpathogenic mycobacterium contamination. Furthermore, the validation results for tuberculosis PCR were questioned.

In serology, the internal and external quality controls were discussed, and some methods were recommended instead of some of sensitive methods like EBV immunblotting. The verifications of ELISA tests were also observed.

The internal and external quality controls are one of the most important points in the accreditation. As advised by the assessor, the three shipments of hepatitis and HIV serology quality controls were then reduced to twice a year. Some tests (E.histolytica antigen ELISA, Quantiferon, etc.) that did not undergo external quality control, were sent to the other accreditated laboratory that has an agreement with our hospital. Hence, the interlaboratory quality controls were also presented to the assessors. The internal controls were also collected in the files to be presented in the assessment.

\section{Verification and validation procedures}

Verification and validation procedures are the most important analyses performed in the laboratory. The literature is insufficient to be understood for applying the standard easily. Here, two of the literatures were analysed and a conflict in terming in home brew tests was observed. Verification is a one-time process performed to confirm the test's expected performance before implementation in the laboratory. Validation is an ongoing process of monitoring a test to ensure that it continuously performs as expected. Therefore, validation is a part of the laboratory quality control programme (Clark et al., 2009). Verification should be performed for a new test or test system for viral antibodies, antigens and nucleic acid. If these tests are home brew tests or test systems, they must be validated (Rabenau et al., 2007). As verification 
contains accuracy, precision and linearity, specificity and sensitivity is included in the validation. Hence, home brew tests require an extensive performance verification; this verification may also be referred to as validation (Clark et al., 2009).

These procedures are mostly applied to quantitative analyses, because the tests are mostly qualitative tests in the microbiology laboratory, which is hard to interprete.

\section{Corrective/preventive action}

There are some complaints and problems in the microbiology laboratory process. Actions are taken to correct or prevent these problems. With regard to external quality controls and internal controls, these corrective actions provided us a great control and self correction. We increased the rate of success in the quality controls. We encouraged our laboratory personnel to fill out the laboratory discrepancy form to control our process and to arrange education. In 2009, among the central laboratories, 60 of the 85 forms were completed in the microbiology laboratory.

The Laboratory Satisfaction Management Procedure is documented in order to define the methods for inspection of proposals, complaints, improvement / correction requests and objections from customers (contracted institutions / doctor / patient), to initiate corrective / protective actions when necessary, monitoring the quality of the test results, effectively achieving improvement of service quality, providing data for improvement and development studies.

The Laboratory Discrepancy Management Procedure provides the basis to start inspection for detecting the main reasons of the problem. Possible reasons, customer requests, patient samples, sample characteristics, methods and procedures, capabilities and training of the personnel, consumption materials and equipment are evaluated under the context of the reason analysis.

When a corrective action is needed in the Central Laboratories of Yeditepe University Hospitals, the possible corrective actions are described. Corrective actions are begun in order to eliminate the factors causing the discrepancies. Corrective actions are based on information like customer complaints, internal audit results, quality records, and the views of employees. Corrective actions are managed according to defined plans by staff that has sufficient qualification.

Selection and application of the most convenient action for solution of the problem and preventing its recurrence is evaluated and followed under the Determination of Discrepancy and Corrective Preventive Action Management Procedure.

\section{Continuous improvement}

Statistical analyses were held throughout a year and this information helped us in making improvements. These are referred to as indicators, and contain statistics about the number of daily test numbers, daily routine culture reporting time, internal quality control results, external quality control results, test results declared to the Ministry of Health, critical value rates, parasitic examination results, unaccepted microbiology specimens (like specimens without identity or those sent by an improper transport container), the antimicrobial resistance, the repeated tests, and the evaluation of the suppliers. For example, while evaluating the kit suppliers, we could change the supplier when the supplier obtains a low grade in the evaluation. We made improvement in the hospital theaters when the number of unaccaptable specimens became significant. A meeting was arranged between the microbiology department and the head nurse in the theater; transport tubes and the microbiology test requisition forms were placed in drawers in every room. Dramatically, the rate decreased. We also improved the rate of timing as $97 \%$ in emergency microbiology tests, such as the rapid antigen tests, hepatitis serology tests and direct stool microscopy.

\section{Education}

Education for laboratory personnel is the main principle during and after accreditation. During the assessment, the number of education hours and the updated textbooks in the laboratory were most appreciated by the assessors. In the test documents, the textbooks and the other reference books were cited in the reference section. The number of education hours in one year was found as $78 \mathrm{~h}$ and $30 \mathrm{~min}$ during the routine laboratory process. During the assessment, the technicians were questioned about the methods, results and interpretation of the tests. The limitations of the tests (like staphylase, oxidase, some test kits like Legionella urinary antigen) were evaluated in detail. The algorithm for the culture and interpretation was also described in the procedure and the education was also given to the technicians in the culture rotation. The personnel was also questioned regarding Oxidase-positive or catalase-positive bacteria.

\section{Hygiene in the laboratory}

As a microbiology laboratory, we have a hygiene plan (stated as daily and monthly). For surfaces and instruments or devices, everyone should know how to perform disinfection and how to clean. The laboratory personnel is educated in using the hand disinfectants. The use of gloves was another important point that the assessor observed during the assessment. We also have a disinfection procedure in exposure of chemical or infectious material. Education of hygiene and disinfection is given to laboratory personnel yearly. Practice about 
fire, laboratory safety and chemical exposure is also trained yearly.

\section{DISCUSSION}

Accreditation is a very hard process and should be tackled with team work. There should be someone responsible to manage the laboratory. Beside the director of the laboratory, a quality director of the hospital should be at work. It is not so easy for us as laboratory personnel to understand the standard itself; however, the quality director also needs someone from the laboratory to apply the standard for the laboratory. The weekly meetings were applied and programmes were arranged in order to complete all the requirements. Sometimes, personnel from other departments of the hospital (biomedical engineering, nursing department, etc.) also attended our meetings.

Our goal is to reduce the laboratory personal errors to nearly zero, to provide continuous education to our personnel and hospital personnel, combining classical methods with new methods, and updating the knowledge with new reference books. Furthermore, we aim to be a reference laboratory in Turkey as an accreditated microbiology laboratory and we wish to share our experience with anyone who wants to take steps in this path. Our microbiology societies such as the Turkish Microbiology Society (TMC) and The Association of Clinical Microbiologists (KLIMUD) should also support this process in our country.

During the assessment (one assessor for microbiology and one assessor for virology) we shared all our knowledge with the assessors and they shared their experience from the other laboratories they had visited.
Improvements were made after mutual talks and discussion.

Hence, accreditation is a continuous and never ending education. We are still rewriting the documents or writing new MSDS as the kits change or to validate new methods, etc.

The experiences should be shared and as a policy, laboratories should acquire accreditation. As a national aim, we should arrange our own quality controls.

\section{REFERENCES}

Amsterdam D, Barenfanger J, Campos J, Cornish N, Daly JA, DellaLatta P, Gray LD, Hall GS, Holmes H, Sautter RL (2004). Cumitech 41: Detection and prevention of clinical mictobiology laboratoryassociated errors. James W Snyder (Co-ordinating ed.). ASM Press,Washington D.C., pp. 2-4.

Clark RB, Lewinski MA, Loeffelholz MJ, Tibbetts RJ (2009). Cumitech 31A: Verification and validation of procedures in the clinical microbiology laboratory. SE Sharp (Coordinating ed.. ASM Press Washington DC., pp. $2-5$.

ISO (2007). Medical laboratories-Particular requirements for quality and competence (ISO 15189). International Organisation for Standardisation (ISO), Geneva.

Kenny D (2001). ISO and CEN documents on quality in medical laboratories. Clin Chim Acta, 309: 121-125.

Rabenau HF, Kessler HH, Kortenbusch M, Steinhorst A, Raggam RB, Berger A (2007). Verification and validation of diagnostic laboratory tests in clinical virology. J. Clin. Virol., 40: 93-98. 